\title{
GASTROINTESTINAL TRACT MULTIPLE LYMPHOMATOUS POLYPOSIS PRESENTED AS MANTLE CELL LYMPHOMA
}

\author{
Fatih Erkan Akay ${ }^{1}$, Nuray Can², Mert Cezik², Hakkı Onur Kırkızlar ${ }^{3}$, Fatma Selin Soyluoğlu ${ }^{4}$, Fulya Öz Puyan ${ }^{2}$ \\ ${ }^{1}$ Trakya University School of Medicine, Edirne, TURKEY \\ ${ }^{2}$ Department of Pathology, Trakya University School of Medicine, Edirne, TURKEY \\ ${ }^{3}$ Division of Hematology, Department of Internal Medicine, Trakya University School of Medicine, Edirne, TURKEY \\ ${ }^{4}$ Department of Nuclear Medicine, Trakya University School of Medicine, Edirne, TURKEY
}

\begin{abstract}
Aims: Mantle cell lymphoma is a mature B cell non-Hodgkin lymphoma which may be presented with the involvement of the gastrointestinal tract as multiple lymphomatous polyposis. The aim of this case report is to increase the awareness of including lymphomatoid polyposis as an entity in the differential diagnosis of multiple polyposis of the gastrointestinal tract. Case Report: A 69-year-old male patient was admitted to the Trakya University Emergency Department with acute abdominal pain. His clinical findings were anorexia that started 3-4 months ago together with constipation and nausea causing him to lose 10-15 kg in 7-8 months, with denial of other parameters of B-symptoms (fevers and night sweats). Endoscopic biopsies that were taken from bulbus and duodenum were investigated and he was diagnosed with mantle cell lymphoma. The patient went through an ileocecal resection due to his intussusception that caused abdominal pain in the first place. Conclusion: Although being an infrequent disease, gastrointestinal lymphomatoid polyposis should be an entity comprised in differential diagnosis for multiple polyposis of the gastrointestinal tract. On the other hand, there is still not a therapeutic protocol with a definitive cure for gastrointestinal tract mantle cell lymphoma. Elderly patients in high risk group such as our patient should be given treatment by taking their conditions into consideration. Keywords: Mantle cell lymphoma, polyp, non-Hodgkin lymphoma
\end{abstract}

\section{INTRODUCTION}

Within large group non-hodgkin lymphomas (NHL) mantle cell lymphoma (MCL) is an infrequent type of B-cell NHL in the World Health Organization (WHO) classification, accounting for nearly $5-10 \%$ of all lymphoid malignancies (1). Primarily occurring in elderly males with a mean age of 65-75 years. MCL has an incidence of 2-3/100.000 per year (1).

Most patients are diagnosed with advanced disease (Ann Arbor stage III/IV) presenting with hepatosplenomegaly, extensive lymphadenopathy, and bone marrow involvement, with some additionally having extranodal involvements including the gastrointestinal (GI) tract, lacrimal glands, central nervous system and skin (2-6). Patients can also exhibit pancytopenia or have a leukemic presentation with massive leukocytosis (4).
Overall, GI tract involvement is the most prevalent extranodal involvement among all lymphomas (5). Mucosal involvement of the small bowel or colon displays itself as a polyp-like lesion (lymphomatoid polyposis) $(5,6)$. Gastrointestinal polyposis, found in up to $10 \%$ of cases, comprising a condition such as multiple lymphomatous polyposis (MLP) is an entity defined by the existence of multiple GI polypoid lesions featured in several segments of the digestive tract $(7,8)$. This case report presents multiple polypoid involvements of the bulbus, ileum, and caecum by aiming to raise awareness of including lymphomatoid polyposis as an entity in the differential diagnosis of multiple polyposis of the GI tract. 


\section{CASE REPORT}

A 69-year-old male patient was admitted to the Trakya University Emergency Department with acute abdominal pain. He had a history of anorexia that started 3-4 months ago together with constipation and nausea causing him to lose 10-15 kg (more than $10 \%$ of total body weight) in 7-8 months. However, he did not have other B symptoms such as fevers and night sweats. The patient also had a medical history of systemic lupus erythematosus and hypertension. He was a 40 pack-year smoker and an occasional consumer of alcohol. He was later administered to General Surgery Department with a pre-diagnosis of intussusception.

On his abdominal magnetic resonance imaging (MRI) he had bilateral pleural effusion, ascites and multiple nodular infiltrations in his spleen and liver. Starting from the antropyloric region of the stomach extending to the first part of the duodenum, a mass lesion forming a prominent expansion in the posterior region was observed. Intussusception and proximal obstruction at the terminal ileum level were observed in the right lateral side of the abdomen. The patient also had a positron emission tomography (PET/CT), revealing extensive lymph node adenopathy throughout his whole body and had a diffuse heterogeneous uptake in his skeletal system, being more in the proximal bilateral humerus (Figure 1, 2).

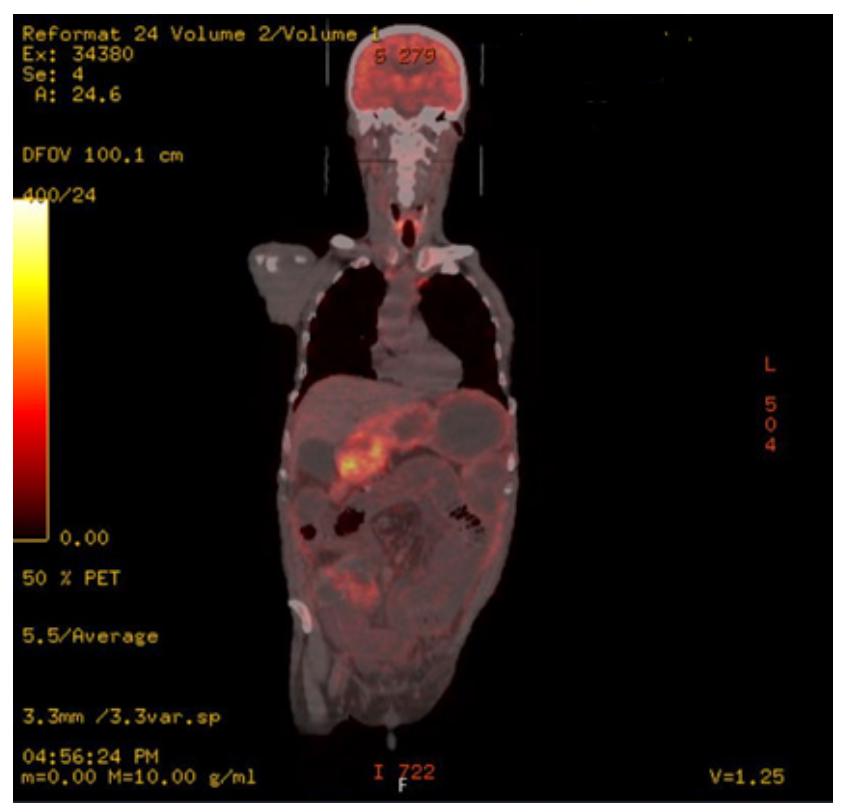

Figure 1: Coronal fused F-18 FDG PET/CT image showing increased FDG uptake throughout the thickened distal gastric wall, in right inferior cervical, bilateral mediastinal and abdominal lymph nodes.

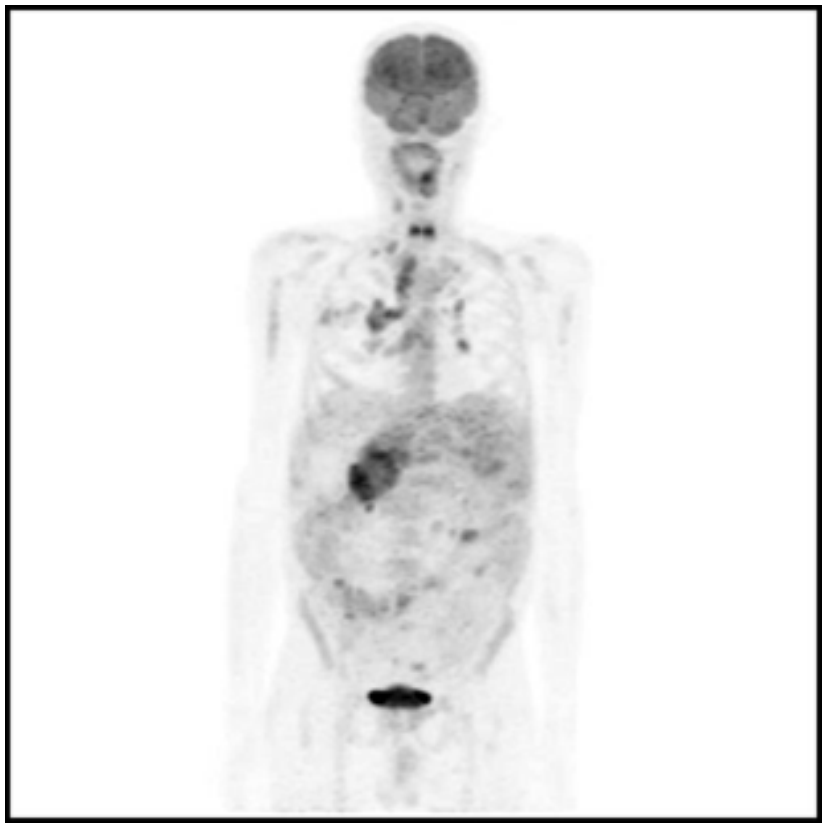

Figure 2: Whole- body MIP (maximum intensity projection) image demonstrating increased FDG uptake in cervical, abdominal and mediastinal lymph nodes, stomach, duodenum, spleen and heterogeneous uptake in the bone marrow.

Endoscopic biopsies that were taken from bulbus and duodenum were investigated by the Pathology department and the patient was diagnosed with MCL. At the same time, the patient urgently went through an ileocecal resection due to his intussusception. Endoscopic samples and resection specimens revealed diffuse infiltration of the intestinal wall by MCL (Figure 3). Immunohistochemical analysis showed positive staining for CD5, CD20, Sox11, and Cyclin D1 and negative for CD10, CD23, blc6, and p53; 70\% of tumor cells were found to be positive for Ki-67 (Figure 4).

After his diagnosis, he was directed to Hematology Clinic for his treatment. On account of his clinical staging, bone marrow biopsy was performed and revealed an infiltration of MCL as well. Bone marrow biopsy revealed minimal involvement of MCL with a less than $10 \%$ positive staining of Cyclin D1 and SOX-11. Patient's MCL International Prognostic Index (MIPI) score was 7.7, which places him into a high-risk category $(9,10)$. According to local and international guidelines, his treatment was planned with autologous stem cell transplantation (ASCT) and high dose chemotherapy. The patient's Eastern Cooperative Oncology Group (ECOG) performance status was 3-4 and cardiac status was low to apply an anthracycline 
based high dose therapy (11).Due to his performance and cardiac status, the patient received rituximab, cyclophosphamide, vincristine, and prednisone (R-CVP) chemoimmunotherapy. It was planned for him to undergo at least three cycles of chemoimmunotherapy with a re-staged evaluation using PET/CT.

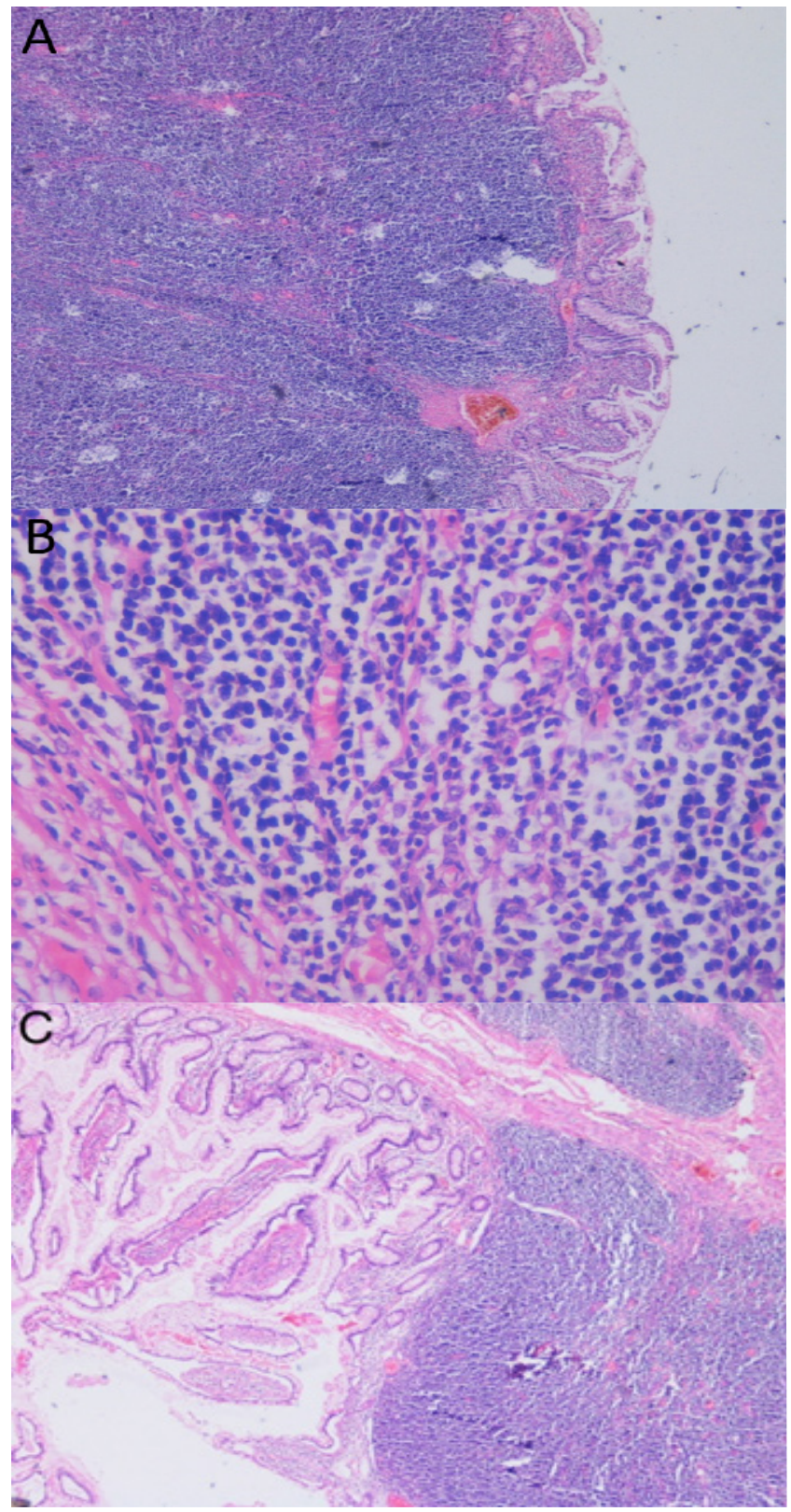

Figure 3: Lymphoid infiltration of tumor cells beneath intestinal mucosa(A: Hematoxyline \& eosin stain, x40, B: Hematoxyline \& eosin stain, x100), transition zone (C: Hematoxyline \& eosin stain, $x 40$ ).

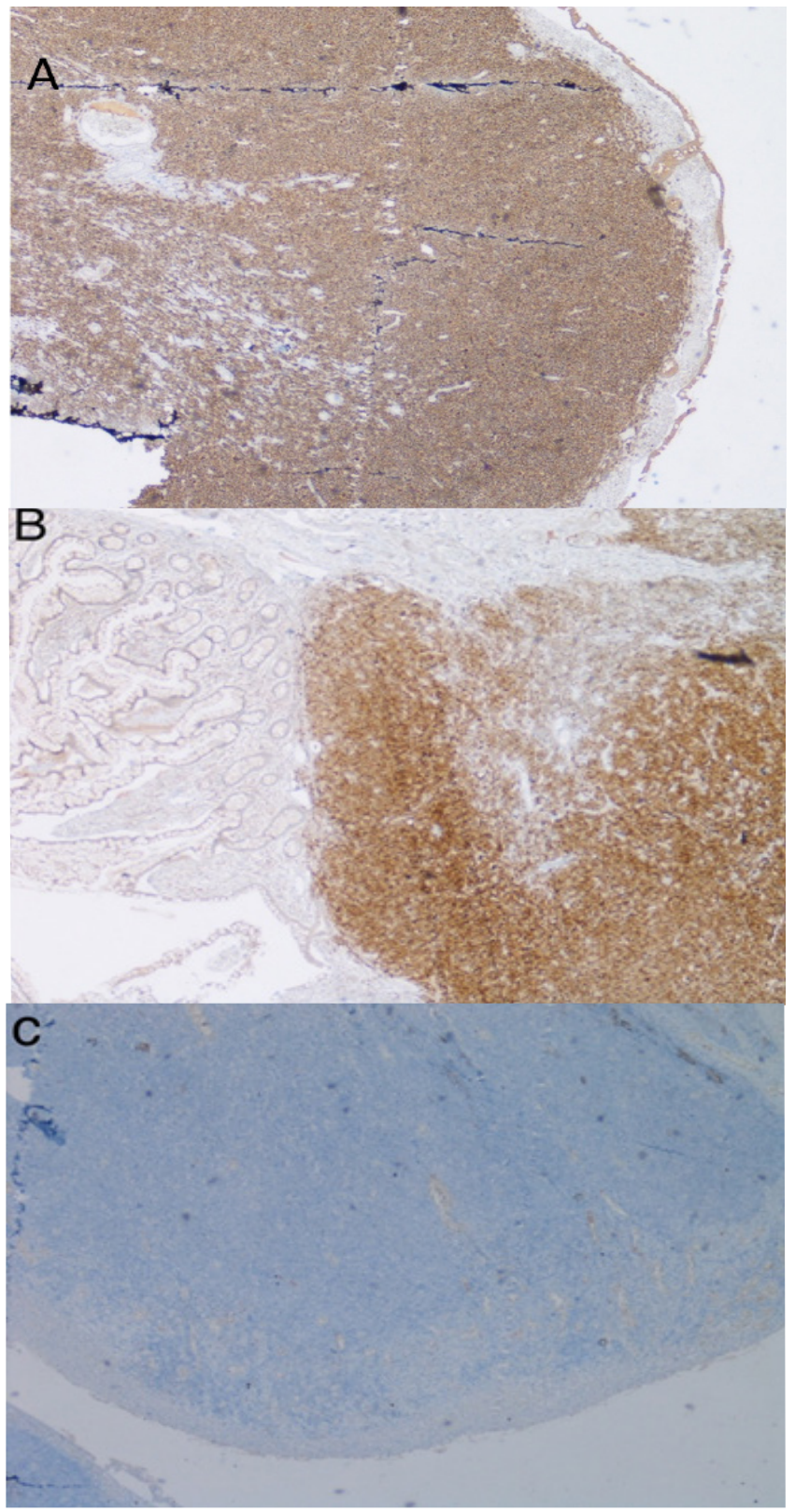

Figure 4: Diffuse positive staining of CD20 (A: Hematoxyline \& eosin stain, $x 40$ ), diffuse positive staining of SOX-11 (B: x40), negative staining of CD23 (C: $x 40)$. 


\section{DISCUSSION}

Multiple lymphomatous polyposis was described by the appearance of diffuse proliferation of atypical lymphocytes accompanied by multiple polypoid lesions throughout GI sites first depicted by Cornes et al. (8) in 1961. The involvement of the GI tract remains ambiguous; however, it has been put forward to be associated with the manifestation of adhesion molecules such as the mucosal homing receptor $\alpha 4 \beta 7(12,13)$.

As the name indicates, malignant cells closely resemble B cells that normally surround the germinal center of the lymph node creating the mantle zone. The molecular pathogenesis is resulting from the chromosomal translocation $\mathrm{t}(11 ; 14)(\mathrm{q} 13 ; \mathrm{q} 32)$ catalyzing deregulation of the $\mathrm{Bcl}-1$ oncogene on chromosome 11, causing overexpression of cyclin D1 (13). Immunohistochemistry is used for differentiating MLP from other NHL, in which malignant cells usually express positivity for CD20, CD5 and Cyclin D1 while being negative for CD3, CD10 and Bcl6. Immunohistochemical analysis of our patient showed positive staining for CD5, CD20, Sox11 and Cyclin D1 whereas negative for CD10, CD23, bcl6 and p53.

Having a heterogeneous clinical picture, the most prevalent symptoms of GI MCL are abdominal pain, hematochezia, and diarrhea $(5,6)$. It should be noted that our patient was admitted to the hospital with acute abdominal pain. The differential diagnosis for GI MCL includes hereditary polyposis syndromes adenomatous polyps, colorectal carcinoma, lymphoid nodular hyperplasia, GI lipomatosis, with hypogammaglobulinemia, among others (8). Mucosa-associated lymphoid tissue lymphoma may present as multiple but has lymphoepithelial lesions and is negative for CD5 and cyclin D1.

International prognostic index risk score for mantle cell lymphoma is currently practiced evaluating patients' prognosis, which encompasses the factors of age, leukocyte count, lactate dehydrogenase level (LDH), performance status, and the percentage of Ki-67 dividing patients into high, intermediate, and low-risk categories $(15,16)$. Our patient was in the high-risk group with a poor prognosis due to his $\mathrm{Ki}-67$ proliferation index of $70 \%$ and MIPI of 7.7. Unfortunately, most patients up to $70 \%$ are diagnosed at stage IV, with a 29-month survival (10). MCL reported overall survival rate is 3-4 years (10).

The present medical method is primarily based on the patient's clinical risk factors, stage of disease and symptoms. Our patient fits the category of elderly or physically unfit patients who are mainly autologous stem-cell transplant-ineligible. Even though the 'gold standard' therapy for young and fit patients (transplant eligible and $<66$ years) is a high dose of chemoimmunotherapy accompanied by ASCT, for the elderly patients ineligible for ASCT, conventional chemoimmunotherapy (e.g. R- CHOP) supported by rituximab, seems to be the 'gold standard' (10). Other agents such as Bendamustine, combined with Rituximab (BR) were also used in elderly group patients, however, one study displays a higher incidence of second cancer in the BR group (19\%) than the R-CHOP group (11\%) (16). Different combinations with agent lenalidomide have been also tested $(16,17)$. Owing to our patients' low cardiac status he was administered a 3-cycle R-CVP chemoimmunotherapy with a re-staged evaluation using PET/ CT.

In conclusion, albeit being an infrequent disease, GI lymphomatoid polyposis should be an entity comprised in the differential diagnosis for multiple polyposis of the GI. On the other hand, there is still not a therapeutic protocol with a definitive cure for GI tract MCL. Elderly patients in high risk group such as our patient should be given treatment by taking their conditions into consideration.

\section{Ethics Committee Approval: N/A}

Informed Consent: Informed consents was obtained from the patient fort his study.

Conflict of Interest: The authors declared no conflict of interest.

Author contributions: Concept: FEA, OPF, KHO. Design: FEA, OPF, KHO. Supervision: FEA, OPF, KHO. Resources: FEA, FOP, HOK, NC, MC, FSS. Materials: FEA, FOP, HOK, NC, MC, FSS. Data collection and/or processing: FEA, FOP, HOK, NC, MC, FSS. Analysis and/or Interpretation: FEA, FOP, HOK, NC, MC, FSS. Literature Search: FEA, FOP, HOK, NC, MC, FSS. Writing Manuscript: FEA, FOP, HOK. Critical Review: FEA, FOP, HOK, NC.

Financial disclosure: The authors declared that this study received no financial support.

Editor-in-chief's Note: One of the authors of this article, Fatih Erkan Akay is a member of the editorial board of Turkish Medical Student Journal. However, he did not take place in any stage on the editorial decision of the manuscript. The editors who evaluated this manuscript are from another institutions.

\section{REFERENCES}

1. Swerdlow SH, Campo E, Harris NL et al. WHO classification of tumours of haematopoietic and lymphoid tissues. Blood 2011;117:5019-32.

2. Freeman C, Berg JW, Cutler SJ. Occurrence and prognosis of extranodal lymphomas. Cancer 1972;29:252-60. 
3. Cheah CY, George A, Gine E et al. Central nervous system involvement in mantle cell lymphoma: clinical features, prognostic factors and outcomes from the European Mantle Cell Lymphoma Network. Ann Oncol 2013;24:2119-23.

4. Ferrer A, Salaverria I, Bosch F et al. Leukemic involvements is a common feature in mantle cell lymphoma. Cancer 2007;109:2473-80. 5. Romaguera JE, Mederious LJ, Hagemeister FB et al. Frequency of gastrointestinal involvement and its clinical significance in mantle cell lymphoma. Cancer 2003;97:586-91.

6. Ruskone-Fourmestraux A, Delmer A, Lavergne A et al. Multiple lymphomatous polyposis of the gastrointestinal tract: prospective clinicopathologic study of 31 cases. Gastroenterology 1997;112:7-16. 7. Dawsey SP, Gregory JA, Brwon AW et al. Asymptomatic multiple lymphomatous polyposis identified during staging bidirectional endoscopy of mantle cell lymphoma. Case Rep Oncol 2016;9(3):661-5. 8. Cornes JS. Multiple lymphomatous polyposis of the gastrointestinal tract. Cancer 1961;14:249-57.

9. Carbone PP, Kaplan HS, Musshoff K et al. Report of the committee on Hodgkin's disease staging classification. Cancer Res 1971;31:18601.

10. Hoster E, Dreyling M, Klapper W et al. A new prognostic index (MIPI) for patients with advanced-stage mantle cell lymphoma. Blood 2008;111:558-65.

11. Oken MM, Creech RH, Tormey DC et al. Toxicity And Response Criteria Of The Eastern Cooperative Oncology Group. Am J Clin Oncol 1982;5:649-55.

12. Drillenburg P, Van der Voort R, Koopman G et al. Preferential expression of the mucosal homing receptor integrin alpha 4 beta 7 in gastrointestinal non-Hodgkin's lymphomas. Am J Pathol 1997;150:919-27.

13. Geissmann F, Ruskoné-Fourmestraux A, Hermine $\mathrm{O}$ et al. Homing receptor alpha 4 beta 7 integrin expression predicts digestive tract involvement in mantle cell lymphoma. Am J Pathol 1998;153:1701-5.

14. Vogt N, Dai B, Erdmann T et al. The molecular pathogenesis of mantle cell lymphoma. Leuk Lymphoma 2017;58(7):1530-7.

15. Geisler $\mathrm{CH}$, Kolstad A, Laurell A et al. Long-term progression-free survival of mantle cell lymphoma after intensive front-line immunochemotherapy with in vivo-purged stem cell rescue: a nonrandomized phase 2 multicenter study by the Nordic Lymphoma Group. Blood 2008;112:2687-93.

16. Albertsson-Lindblad A, Kolstad A, Laurell A et al. Lenalidomide-bendamustine-rituximab in patients older than 65 years with untreated mantle cell lymphoma. Blood 2016;128:1814-20.

17. Gressin R, Daguindau N, Tempescul A et al. A phase 2 study of rituximab, bendamustine, bortezomib and dexamethasone for first-line treatment of older patients with mantle cell lymphoma. Haematologica 2019;104:138-46. 